\title{
Visualization of liver lesions in standardized video-documented ultrasonography - inter-observer agreement and effect of contrast injection.
}

\section{Carina Stenman ${ }^{1,2}$, Robert Glavas', Joachim Davidsson'1, Anders Knutsson'1, Örjan Smedby ${ }^{1,2}$}

${ }^{1}$ Department of Radiology and Department of Medical and Health Sciences, ${ }^{2}$ Center for Medical Image Science and Visualization (CMIV), Linköping University, Linköping, Sweden.

\begin{abstract}
The aim of this study was to evaluate the inter-observer agreement and effect of contrast injection on the visibility of liver lesions by radiologists reviewing ultrasound examinations acquired by a radiographer using a standardized examination protocol. Material and method: A retrospective review was conducted by two radiologists, independently of each other, of 115 ultrasound examinations of the liver with standardized examination protocols between January 2008 and December 2012. All patients included in the study had undergone surgery for colorectal cancer. Patients attending the two-year follow-up were included. Results: Focal findings, the most common of which were cysts, were seen in $42-43$ out of the 115 patients before intravenous contrast and in 46-47 patients after intravenous contrast $(p=0.012)$. The inter-observer agreement for focal findings was $86.1 \%$ before contrast, and $90.4 \%$ after contrast (n.s.), and the corresponding kappa values were 0.72 and 0.84 , respectively. Conclusion: A good inter-observer agreement between two radiologists reviewing ultrasound examinations (standardized ultrasound cine-loop method acquired by a radiographer) after surgery for colorectal cancer was obtained. Injection of contrast medium increased the visibility of liver lesions.
\end{abstract}

Keywords: liver, contrast-enhanced ultrasound, inter-observer agreement, standardized examination

\section{Introduction}

Metastases from colorectal cancer develop in approximately $50 \%$ of patients [1]. To increase the chances of cure, it is important to discover the metastases at an early stage, and the diagnosis relies on imaging [1]. Imaging methods that may be used include ultrasound, Magnetic Resonance Imaging (MRI) and Computed Tomography (CT). The examination method to be used depends on local equipment, availability and operator expertise [1]. Contrast-enhanced ultrasonography (CEUS) has im-

Received 15.05.2015 Accepted 13.08.2015

Med Ultrason

2015, Vol. 17, No 4, 437-443

Corresponding author: Carina Stenman

Department of Radiology and Department of Medical and Health Sciences

Linköping University Hospital, Garnisonsvägen, 58185 Linköping, Sweden

Fax: 0046101031799

E-mail: carina.stenman@liu.se proved both the detection and characterization of focal liver lesions and is a useful imaging method for distinguishing benign from malignant liver lesions [2,3]. There are three vascular phases, the late phase being the most important phase for distinguishing benign from malignant lesions, the hypovascularisation in this phase being the most specific signs of malignancy $[4,5]$. Previous studies have shown that CEUS is an accurate imaging method for the characterization of focal liver lesions and could be compared with contrast-CT and contrast MRI [6]. CEUS using a second-generation micro-bubble contrast agent poses no risk of nephrotoxicity and requires no radiation. It is possible to visually assess the liver perfusion throughout the vascular phases in real time [4].

Today, the most common way to document ultrasound examinations is to store static images from regions of interest or where pathology is seen. This method offers very limited possibilities for the re-evaluation of an ultrasound examination. Although the patient may have been examined in a systematic way, only the examiner knows 
what is seen before and after the static images. Therefore, this is usually considered as an operator-dependent method. The value of re-evaluating static images is thus very limited, and the experience and education of the examiner are crucial when using this method.

Examinations using standardized scanning protocols, documented with cine-loops and stored in a dedicated PACS, may be a solution $[7,8]$. The standardized scanning protocol includes both longitudinal and transversal views covering the whole organ or region of interest [8]. After the examination, the cine-loops are stored in the PACS, and the evaluation is made later at dedicated workstations, where the examination can be viewed once or many times. It is recommended that essential clips for each vascular phase should be recorded [3].

It has been shown that with the use of this standardized technique in abdominal ultrasound, there is high accuracy and a high inter-observer agreement between bedside and offline reading [9]. One advantage of this approach is that the examinations may be performed by a radiographer and evaluated later by a radiologist, thus releasing more of the radiologist work force [10], and increasing the availability of radiologists for acute or more complex examinations.

The purpose of this study was to evaluate the visibility of lesions and the inter-observer agreement between radiologists reviewing ultrasound examinations of the liver with unenhanced ultrasound and CEUS, when the examination is performed by a radiographer, using a standardized examination protocol.

\section{Material and method}

A retrospective review was performed of 115 ultrasound examinations of the liver before and after a contrast injection between January 2008 and December 2012 from our center. The subjects were 62 males and 53 females, mean age 73 , range 46-93 years old. The study was approved by the local Ethics Committee. Patients included in the study had undergone surgery for colorectal cancer. According to the clinical routine at our hospital, CEUS is performed from 6 month to 3 years with six-month intervals after surgery to evaluate the liver with the clinical question of metastases. In the current study, a consecutive material was collected, consisting of all patients who came for the two-year follow-up. The patients were fasting 6 hours prior to the examination. The sonographic examination was carried out by a radiographer with the use of the standardized ultrasound cine-loop method, i.e. films covering 5-10 centimeters in 5-10 seconds [11]. All data were acquired by the same radiographer, using a scanning protocol for the liver and biliary system con- sisting of approximately 7 to 11 loops. The segments in the liver were scanned first transversal and then longitudinal. Always in the direction from cranial to caudal and from left to right, independent of patient position. The goal of the examination was to cover the entire liver and identify the segments where any pathology was located. For segment 2 and 3 the patient lay in the supine position, and for segment 1 and 4-8 the patient lay in the left decubitus position [8]. The dynamic films were stored in a dedicated PACS (Syngo Dynamics, Siemens Medical Systems, Erlangen, Germany), and evaluation was made later at dedicated workstations permitting the examination to be viewed once or many times for writing the report. The radiographer had worked with ultrasound for about 6 years, consistently using this standardized method. All sonograms were obtained with ACUSON Sequoia (Siemens Medical Systems, Erlangen, Germany), using a convex transducer $4 \mathrm{C} 1$ with 3 to $4 \mathrm{MHz}$. First, the liver was examined without the intravenous contrast agent using the standardized ultrasound method. After the injection of the contrast agent ( $2.4 \mathrm{ml}$ of SonoVue, Bracco, Italy) via a 20 -gauge intravenous catheter placed in a vein and followed by $5-10 \mathrm{ml}$ saline flush, the examination was repeated by the same radiographer beginning 90 seconds from the start of the injection (using a timer starting when the agent was injected), i.e. in the portal and late phase. If a focal lesion was seen before the contrast injection, the acquisition with contrast was used for characterization of the lesion in the arterial phase. The mechanical index was as low as possible, mostly varying between 0.09 and 0.18 , unless a higher value was necessitated by the size of the patient or by steatosis. Ultrasound parameters such as gain adjustment, focal zone locations and depth were changed on a case-by-case basis throughout the examination. Each examination lasted at most 4.5 minutes after the injection.

The examinations were reviewed by two radiologists using workstations with identical conditions such as lightning and resolution. The examinations without and with intravenous contrast were reviewed immediately after each other as a matching pair. The radiologists were familiar with the standardized examination protocol, had worked with the method since 2004, and had 8-20 years of experience of abdominal ultrasound. They were also familiar with cine-loop documentation and with reviewing an examination made by someone else. After each review, the radiologists filled out an evaluation form including corresponding data before and after the intravenous contrast. The radiologists were blinded to each other's assessment.

The findings recorded included whether the examination was diagnostic or not, focal changes (classified as cyst, metastases and other focal changes), localization (Couin- 
aud segments) and the number and size [12]. The reviewer was also asked whether he judged the patient to require an additional examination such as MRI or CT. The form contained no patient data. In cases where either of the observer noted a metastases, the medical records were reviewed for histopathological diagnoses or subsequent examination with CT or MRI for verification of the metastases.

\section{Statistical analysis}

Agreement between observers was assessed as percent agreement and kappa statistics [13]. Conditional logistic regression was used to compare frequencies of reported findings between observers and between examination before and after intravenous contrast [14]. The number and size of focal findings in those patients where they were reported were analyzed using mixed-effects analysis of variance. All analyses were carried out in Stata 13.1 (Stata Corp, College Station, TX, USA).

\section{Results}

Out of the 115 patients examined, 112-113 were judged to be diagnostic (table I). The most common finding was cysts, with a frequency of $29-30 \%$ before and $31-32 \%$ after contrast injection. Metastases were reported in 5-6\% before and $9-10 \%$ after contrast injection. In 12 cases where at least one observer noted a metastasis in at least one of the examinations, the finding of metastasis was confirmed with $\mathrm{CT}$ within the next 10 months after the ultrasound examination. In 11 of the 12 cases, the CT examination took place within one month. Five of the patients were deceased. No histopathological diagnoses could be found in the patients' medical record. The difference in frequency was significant for all focal lesions, for metastases, and for cysts, but not for "other focal findings". The other most common finding was steatosis. In no case significant systematic difference between the two observers was found. Three examples of the varying effect of contrast injection are given in figures 1-3.

The frequency of focal findings varied considerably between the eight Couinaud segments, with the highest frequency in segment 6 and the lowest in segment 1 (ta- ble II). The number of patients with pathological findings was significantly higher with CEUS than before contrast injection. With one exception (segment 5), no significant systematic difference between observers was noted.

After injection of the contrast agent, a small but significant increase in the number of reported lesions was noted (table III). The size of the focal lesions did not differ between examinations before and after contrast injection, nor between the two radiologists' assessments.

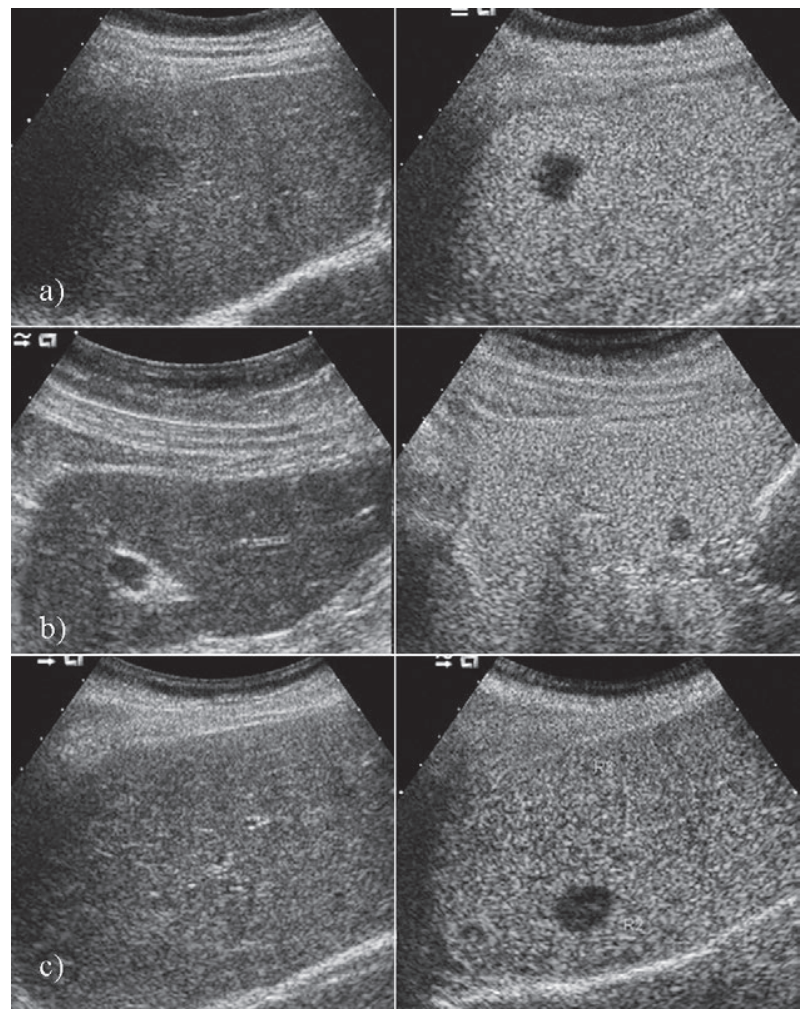

Fig 1. a) The metastasis is seen before contrast injection in the right liver lobe (left image ) but more conspicuous after injection (right image ); b) The metastasis is hardly visible before contrast in the left liver lobe (left image ) and becomes clearly visible after intravenous contrast (right image ); c) The metastasis is not visible before contrast injection in the right liver lobe (left image). After contrast injection a metastasis is seen in segment 7 (right image ).

Table I. Number of patients with diagnostic examinations, positive focal findings and need for further examination without and with intravenous contrast medium $(\mathrm{n}=115)$. Effect of intravenous contrast and observer evaluated with conditional logistic regression.

\begin{tabular}{|c|c|c|c|c|c|c|}
\hline & \multicolumn{2}{|c|}{ Without CEUS } & \multicolumn{2}{|l|}{ With CEUS } & \multicolumn{2}{|c|}{ Significance levels } \\
\hline & Observer A & Observer B & Observer A & Observer B & Contrast & Observer \\
\hline Diagnostic examination & $112(97 \%)$ & $112(97 \%)$ & $113(98 \%)$ & $112(97 \%)$ & $\mathrm{p}=0.597$ & $\mathrm{p}=0.783$ \\
\hline Focal findings & $43(37 \%)$ & $42(37 \%)$ & $47(41 \%)$ & $46(40 \%)$ & $\mathrm{p}=\mathbf{0 . 0 1 2}$ & $\mathrm{p}=0.691$ \\
\hline Metastasis & $6(5 \%)$ & $7(6 \%)$ & $10(9 \%)$ & $12(10 \%)$ & $p=0.021$ & $\mathrm{p}=0.417$ \\
\hline Cyst & $34(30 \%)$ & $33(29 \%)$ & $37(32 \%)$ & $36(31 \%)$ & $p=0.018$ & $\mathrm{p}=0.747$ \\
\hline Other focal finding & $6(5 \%)$ & $5(4 \%)$ & $3(3 \%)$ & $5(4 \%)$ & $\mathrm{p}=0.336$ & $\mathrm{p}=0.817$ \\
\hline Need for further examination & $6(5 \%)$ & $7(6 \%)$ & $5(4 \%)$ & $16(14 \%)$ & $\mathrm{p}=\mathbf{0 . 0 3 0}$ & $\mathrm{p}=0.052$ \\
\hline
\end{tabular}


Table II. Number of liver segments with positive focal findings without and with intravenous contrast medium. Effect of intravenous contrast and observer evaluated with conditional logistic regression.

\begin{tabular}{|c|c|c|c|c|c|c|}
\hline & \multicolumn{2}{|c|}{ Without intravenous contrast } & \multicolumn{2}{|c|}{ With intravenous contrast } & \multicolumn{2}{|c|}{ Significance levels } \\
\hline & Observer A & Observer B & Observer A & Observer B & Contrast & Observer \\
\hline All segments $(n=920)$ & $85(9.2 \%)$ & $93(10.1 \%)$ & $102(11.1 \%)$ & $108(11.7 \%)$ & $\mathrm{p}<0.001$ & $p=0.212$ \\
\hline segment $1(n=115)$ & $1(0.9 \%)$ & $0(0.0 \%)$ & $1(0.9 \%)$ & $0(0.0 \%)$ & - & - \\
\hline segment $2(n=115)$ & $13(11.3 \%)$ & $15(13.0 \%)$ & $14(12.2 \%)$ & $17(14.8 \%)$ & $\mathrm{p}=0.183$ & $p=0.396$ \\
\hline segment $3(n=115)$ & $14(12.2 \%)$ & $10(8.7 \%)$ & $16(13.9 \%)$ & $14(12.2 \%)$ & $\mathrm{p}=\mathbf{0 . 0 3 4}$ & $p=0.240$ \\
\hline segment $4(n=115)$ & $10(8.7 \%)$ & $15(13.0 \%)$ & $17(14.8 \%)$ & $19(16.5 \%)$ & $\mathrm{p}<0.001$ & $p=0.210$ \\
\hline segment $5(n=115)$ & $5(4.4 \%)$ & $10(8.7 \%)$ & $5(4.4 \%)$ & $10(8.7 \%)$ & $\mathrm{p}=1.000$ & $p=0.041$ \\
\hline segment $6(n=115)$ & $17(14.8 \%)$ & $16(13.9 \%)$ & $20(17.4 \%)$ & $17(14.8 \%)$ & $\mathrm{p}=\mathbf{0 . 0 4 4}$ & $p=0.503$ \\
\hline segment $7(n=115)$ & $10(8.7 \%)$ & $13(11.3 \%)$ & $11(9.6 \%)$ & $11(9.6 \%)$ & $\mathrm{p}=0.723$ & $p=0.585$ \\
\hline segment $8(n=115)$ & $15(13.0 \%)$ & $14(12.2 \%)$ & $18(15.7 \%)$ & $20(17.4 \%)$ & $\mathrm{p}=\mathbf{0 . 0 2 2}$ & $p=0.889$ \\
\hline
\end{tabular}

Table III. Size and number of focal lesions without and with intravenous contrast. Effect of intravenous contrast evaluated with mixed-effects analysis of variance.

\begin{tabular}{|c|c|c|c|c|c|c|}
\hline & \multicolumn{2}{|c|}{ Without intravenous contrast } & \multicolumn{2}{|c|}{ With intravenous contrast } & \multicolumn{2}{|c|}{ Significance levels } \\
\hline & $\begin{array}{l}\text { Observer A } \\
(n=43)\end{array}$ & $\begin{array}{l}\text { Observer B } \\
(n=42)\end{array}$ & $\begin{array}{l}\text { Observer A } \\
(n=47)\end{array}$ & $\begin{array}{l}\begin{array}{l}\text { Observer B } \\
(n=46)\end{array} \\
\end{array}$ & Contrast & Observer \\
\hline Number of lesions, mean (SD) & $2.3(1.5)$ & $2.3(1.6)$ & $2.4(1.5)$ & $2.6(1.6)$ & $\mathrm{p}=\mathbf{0 . 0 0 5}$ & $p=0.266$ \\
\hline Lesion diameter, mean (SD) (mm) & $19(15)$ & $19(13)$ & $18(15)$ & $18(13)$ & $\mathrm{p}=0.641$ & $p=0.635$ \\
\hline
\end{tabular}

Table IV. Agreement between observers without and with intravenous contrast $(n=115)$. Effect of intravenous contrast on agreement in percent evaluated with conditional logistic regression.

\begin{tabular}{llllll}
\hline & \multicolumn{2}{c}{$\begin{array}{l}\text { Without intravenous contrast } \\
\text { Agreement (\%) }\end{array}$} & \multicolumn{2}{l}{$\begin{array}{l}\text { With intravenous contrast } \\
\text { Agreement (\%) }\end{array}$} & \multicolumn{2}{c}{ Significance level } \\
& kappa & & \\
\hline Diagnostic examination & 96.5 & 0.316 & 97.4 & 0.387 & $\mathrm{p}=0.644$ \\
Focal findings & 86.1 & 0.719 & 90.4 & 0.836 & $\mathrm{p}=0.141$ \\
Metastasis & 97.4 & 0.756 & 94.8 & 0.699 & $\mathrm{p}=0.311$ \\
Cyst & 90.4 & 0.768 & 92.2 & 0.819 & $\mathrm{p}=0.410$ \\
Other focal finding & 92.2 & 0.141 & 96.5 & 0.483 & $\mathrm{p}=0.141$ \\
Need for further examination & 92.2 & 0.267 & 87.0 & 0.235 & $\mathrm{p}=0.115$ \\
\hline
\end{tabular}

In general, there was good agreement $(90 \%-97 \%)$ between the observers before and after contrast injection (table IV). Two exceptions were focal findings before contrast injection and the need for further examination after contrast injection. When the agreement was evaluated with the kappa coefficient, on the other hand, poor or moderate agreement was found for those questions (diagnostic examination, other focal findings, and need for further examination) where the frequency of positive answers was either below $10 \%$ or above $90 \%$. For the remaining questions (focal findings, metastases, and cysts), the kappa values were 0.70 or higher.

\section{Discussions}

The radiologists in this study observed focal findings in slightly more cases with CEUS than with unenhanced ultrasound when a retrospective review was made of stored cine-loops. As for the inter-observer agreement, the radiologists were fairly consistent in their assessment, but no effect of contrast injection was found.

It is often asserted that standardized and adequate training is needed to obtain optimal and reproducible results from ultrasound examinations, including CEUS [4]. For a complete examination, it is important with a detailed written protocol to establish the exact images that constitute a comprehensive examination [15]. According to previous research, the value of reviewing static ultrasound images of the abdomen is very limited [16]. A study by Faschingbauer et al compared the diagnostic performance in a group of examiner's with four different levels of experience in gynecological ultrasound. Their study showed that interpreting static ultrasound images significantly improved with an increasing level of experience [17]. In other areas where ultrasound is used, it has been found that experience 
plays a major role in the detection of small congenital heart defects [18].

In many institutions, the ultrasound examination is performed by a radiologist, who judges the presence of metastases and other focal findings during dynamic visualization of the liver, usually not following a thoroughly standardized scanning protocol [19]. We have seen an advantage when examining in a standardized way, as it is possible to compare the examination before and after contrast injection in identical views. This standardized technique and the use of an organ scheme is helpful for making even small findings.

In the study by Quaia et al, they retrospectively reviewed cine-loops of liver tumors with CEUS, and the reviewers had 1 to 10 years of experience of abdominal ultrasound. They showed that the diagnostic performance when reviewing cine-loops of CEUS in liver tumor characterization was dependent on the observer's level of experience [20].

According to current recommendations, essential clips for each vascular phase should be recorded [3], and this is also common practice when performing CEUS. Several previous studies show that CEUS is an accurate imaging method for characterization of focal liver lesions $[1,2,4-6,21]$. What we suggest in this study is the standardization of examinations before and after contrast injection. This consistent and systematic way of acquiring image data might potentially increase the sensitivity of the method.

When cine-loops are reviewed at a workstation after the examination, the ability to repeat part of the loop several times may increase the chance of seeing small lesions which might be overlooked at the bedside examination in the examination room, in particular with less experienced examiners. In the study by Jandzinski et al, an increased detection rate was noted for small renal cysts when the examiners used cine-loop documentation and had the possibility to review the entire examination at a workstation [22].

One of the advantages of the standardized method and documented cine-loops is the possibility for a second opinion by a more experienced radiologist, which helps in maintaining a high level of expertise in ultrasound examinations of the abdomen.

The opportunity to review the entire organ may permit the examination to be performed by a radiographer who has learned the technique of visualizing the organ from different angles, giving the radiologist optimal views to make a correct diagnosis. In the study by Gaarder et al, it was shown that standardized ultrasound examinations of kidneys acquired by a trained radiographer have a similar diagnostic value as when the examination is performed by a radiologist [10]. There are previous studies showing good results when comparing examinations by radiographers and radiologists in routine abdominal ultrasound $[23,24]$. Most of these studies have involved general upper abdominal scans. According to Leslie et al, radiographers are as competent as radiologists at performing routine abdominal ultrasound examinations [23].

Bude et al recommended that if the examination is to be performed by a trained sonographer, video documentation should be used [25]. The video clips made it possible to review the examinations several times, and they did not need to call back any patients for a repeat examination. It was concluded that this technique increases the quality and safety for patients in an ultrasound examination. Dormagen et al studied the usefulness of this standardized cine-loop technique when examining the liver, gallbladder, pancreas, kidneys, and urine bladder [9]. They found high accuracy and high inter-observer agreement in the bedside and offline reading made by different radiologists, which shows the diagnostic safety of this technique when reviewing ultrasound examinations at a workstation.

In our study, some of the examinations were suboptimal and an additional examination was recommended due to the liver location, high up under the right arch, in particular when the patient was overweight, which may limit the patient's ability to take a deep breath so that sub-diaphragmal parts of the liver are difficult to visualize. Steatosis also complicates the examination considerably, since the ultrasound penetration to deeper parts of the liver is limited. In the present study, there were 2-3 patients with steatosis whose examinations were judged as not diagnostic. For the examination to be diagnostic it has to cover the entire liver and the cine-loops have to cover at least 5-10 centimeters in 5-10 seconds. If the scanning is faster than 5-10 seconds, important pathology may be missed. Nevertheless, except for patients with severe steatosis, CEUS seems to have potential as a standard technique for detection of liver metastases. In some cases, the metastases were only seen after the contrast injection. In these cases the value of contrast is considerable.

When our two radiologists reviewed the examinations, they did not always use the same workstation, but the reviewing conditions were similar for both reviewers. When reviewing the examinations, the radiologists had no clinical information or history of the patient. Both radiologists had the possibility to review the examination several times and thus reevaluate their assessment.

The reviewers in our study had worked closely together for several years and may thus have influenced each other's practical skills. When generalizing to other 
settings, it is therefore not certain whether equally high inter-observer agreements can be expected. In several cases, the two radiologists did not agree on what specific segment of the liver the focal lesions belonged to due to the localization of the lesion close to the boundary between two segments. Normal variation in patient anatomy may also complicate the assessment of the exact location. In addition, the reader's experience from ultrasound may influence the diagnostic assessment. It may be noted that observer A, who had 20 years of experience, indicated a need for further examination after intravenous contrast in only 5 cases, whereas observer B, with 8 years, did so in 16 cases.

A more severe limitation, however, was the lack of a reference method such as CT or MRI for evaluating this standardized ultrasound method. In future studies, it would be desirable to assess the diagnostic accuracy of the standardized technique by correlating it to a reference standard. If the diagnostic accuracy is satisfactory, the widespread availability and lack of ionizing radiation make CEUS with standardized technique a very attractive choice for follow-up in patients who have undergone surgery for colorectal cancer.

This standardized technique, with a radiographer performing the acquisition, may improve the workflow, while the diagnostic accuracy may be enhanced by the increased possibilities of obtaining a second opinion after the examination. Ultrasound then becomes more similar to other radiological modalities such as CT and MRI, where standardized examination protocols are used. With this technique, the imaging is less operator-dependent, since the entire liver is imaged with a standardized scanning protocol. Another advantage is the potential for the education of new radiologists, since it is possible to overview the entire ultrasound examination at the workstation along with an experienced radiologist.

\section{Conclusions}

Overall, we found a good agreement between the two radiologists reviewing CEUS liver examinations, acquired by a radiographer using a standardized technique, after surgery for colorectal cancer. Injection of contrast medium increased the visibility of liver lesions.

\section{Conflict of interest: none}

\section{References}

1. Xu LH, Cai SJ, Cai GX, Peng WJ. Imaging diagnosis of colorectal liver metastases. World J Gastroenterol 2011; 17: 4654-4659.
2. Danila M, Popescu A, Sirli R, Sporea I, Martie A, Sendroiu M. Contrast enhanced ultrasound (CEUS) in the evaluation of liver metastases. Med Ultrason 2010; 12: 233-237.

3. Claudon M, Dietrich CF, Choi BI, et al. Guidelines and good clinical practice recommendations for Contrast Enhanced Ultrasound (CEUS) in the liver - update 2012: A WFUMB-EFSUMB initiative in cooperation with representatives of AFSUMB, AIUM, ASUM, FLAUS and ICUS. Ultrasound Med Biol 2013; 39: 187-210.

4. Jang JY, Kim MY, Jeong SW, et al. Current consensus and guidelines of contrast enhanced ultrasound for the characterization of focal liver lesions. Clin Mol Hepatol 2013; 19: $1-16$.

5. Nicolau C, Ripolles T. Contrast-enhanced ultrasound in abdominal imaging. Abdom Imaging 2012; 37 :1-19.

6. Sirli R, Sporea I, Martie A, Popescu A, Danila M. Contrast enhanced ultrasound in focal liver lesions--a cost efficiency study. Med Ultrason 2010; 12: 280-285.

7. Stenman C, Thorelius L, Knutsson A, Smedby O. Radiographer-acquired and radiologist-reviewed ultrasound examination--agreement with radiologist's bedside evaluation. Acta Radiol 2011; 52: 70-74.

8. Thorelius L. Sonodynamics fundamentals 2014 [cited 2014, 27 February]. Available from: www.sonodynamics.com.

9. Dormagen JB, Gaarder M, Drolsum A. Standardized cineloop documentation in abdominal ultrasound facilitates offline image interpretation. Acta Radiol 2015; 56: 3-9.

10. Gaarder M, Seierstad T, Soreng R, Drolsum A, Begum K, Dormagen JB. Standardized cine-loop documentation in renal ultrasound facilitates skill-mix between radiographer and radiologist. Acta Radiol 2015; 56: 368-373 .

11. Stenman C, Jamil S, Thorelius L, Knutsson A, Smedby O. Do radiologists agree on findings in radiographer-acquired sonographic examinations? J Ultrasound Med 2013; 32: 513-518

12. Dietrich CF, Serra C, Jedrzejczyk M. Ultrasound of the liver. Bad Mergentheim 2010.

13. Landis JR, Koch GG. The measurement of observer agreement for categorical data. Biometrics 1977; 33: 159-174.

14. Breslow NE, Day NE. Statistical Methods in Cancer Research. Vol. 1, The Analysis of Case-control Studies. (IARC Scientific Publications No. 32), International Agency for Research on Cancer, Lyon 1980.

15. Robbin ML, Lockhart ME, Weber TM, et al. Ultrasound quality and efficiency: how to make your practice flourish. J Ultrasound Med 2011; 30: 739-743.

16. Rosen MP, Levine D, Carpenter JM, et al. Diagnostic accuracy with US: remote radiologists' versus on-site radiologists' interpretations. Radiology 1999; 210: 733-736.

17. Faschingbauer F, Benz M, Haberle L, et al. Subjective assessment of ovarian masses using pattern recognition: the impact of experience on diagnostic performance and interobserver variability. Arch Gynecol Obstet 2012; 285: 16631669.

18. Tegnander E, Eik-Nes SH. The examiner's ultrasound experience has a significant impact on the detection rate of 
congenital heart defects at the second-trimester fetal examination. Ultrasound Obstet Gynecol 2006; 28: 8-14.

19. Piscaglia F, Corradi F, Mancini M, et al. Real time contrast enhanced ultrasonography in detection of liver metastases from gastrointestinal cancer. BMC Cancer 2007; 7: 171.

20. Quaia E, Alaimo V, Baratella E, et al. Effect of observer experience in the differentiation between benign and malignant liver tumors after ultrasound contrast agent injection. J Ultrasound Med 2010; 29: 25-36.

21. Ding H, Wang WP, Huang BJ, et al. Imaging of focal liver lesions: low-mechanical-index real-time ultrasonography with SonoVue. J Ultrasound Med 2005; 24: 285-297.

22. Jandzinski D, van Wijngaarden E, Dogra V, Fisher SG, Conde A, Rubens D. Renal sonography with 2-dimensional versus cine organ imaging: preliminary results. J Ultrasound Med 2007; 26: 635-644.

23. Leslie A, Lockyer H, Virjee JP. Who should be performing routine abdominal ultrasound? A prospective double-blind study comparing the accuracy of radiologist and radiographer. Clin Radiol 2000; 55: 606-609.

24. Lo RH, Chan PP, Chan LP, Wilde CC, Pant R. Routine abdominal and pelvic ultrasound examinations: an audit comparing radiographers and radiologists. Ann Acad Med Singapore 2003; 32: 126-128.

25. Bude RO, Fatchett JP, Lechtanski TA. The use of additionally trained sonographers as ultrasound practitioners: our first-year experience. J Ultrasound Med 2006; 25: 321327. 